\title{
Mut zur Lücke? Flucht vor dem Recht durch bewusste staatliche Nicht-Regulierung
}

\author{
Sarah Katharina Stein
}

Abstract: Staaten können eine Rechtsbindung umgehen, indem sie gar nicht erst das Recht erschaffen, welches sie oder andere binden könnte. Von dieser Möglichkeit der Flucht vor dem Recht haben Staaten im Bereich der Ausübung von Staatsgewalt durch die Nicht-Regulierung von privaten Militär- und Sicherheitsdienstleistern Gebrauch gemacht. Als Ausgleich wurden stattdessen Selbstregulierungsregime erschaffen, die die Handlungen von privaten Militärdienstleistern begrenzen und Schutz für Betroffene herstellen sollen. Diese Selbstregulierungsmechanismen können aber die Einhaltung des humanitären Völkerrechts und der Menschenrechte nicht absichern. Vielmehr perpetuieren sie durch ibre Existenz die regulierungsarme Situation. Opfern von Schädigungshandlungen wird ein Zugang zu ihren Rechten somit vereitelt. Dadurch entstehen Verschiebungen in den Machtverhältnissen zwischen Militärdienstleistern, Opfern von Schädigungshandlungen und Staaten.

Eine „Flucht vor dem Recht“ beschreibt staatliche Umgehungsversuche vor bestehenden Regeln und Pflichten. Dieser Beitrag widmet sich einer speziellen Ausprägung dieses Phänomens: der verweigerten Rechtsetzung durch Staaten. Dabei flüchten Staaten nicht vor bestehendem Recht, sondern erschaffen erst gar nicht das Recht, welches sie oder andere binden könnte. Sie verhindern damit letztlich den Zugang zu Recht, da sich Betroffene nicht auf etwas berufen können, was nicht existiert.

Dieses Phänomen tritt im Kontext der Ausübung von Staatsgewalt auf. Private Militär- und Sicherheitsdienstleister werden von Staaten u.a. in bewaffneten Konflikten zur Ausübung von Gewalt eingesetzt. Im Gegensatz zu anderen Privatisierungsbestrebungen gehen mit dem Outsourcing in diesem Falle aber keine Regulierungsanstrengungen einher, da sich staatliche Anreize nur dann bewähren, wenn die Branche nicht gesetzlich gesteuert wird. In dieser Regulierungslücke sind zunehmend Selbstregulierungsbemühungen zu finden. Diese können jedoch keine effektive Überantwortung von Rechten und Pflichten auf militärische Dienstleister bewirken. Dadurch entstehen Verschiebungen in den Machtverhältnissen 
zwischen Militärdienstleistern, Opfern von Schädigungshandlungen und Staaten.

\section{Private Militärdienstleister als globale Akteure}

\section{Private Militärfirmen}

Private Militärfirmen (private military companies, PMCs) sind privatwirtschaftlich organisierte Unternehmen, die verschiedene Sicherheits- und Militärdienstleistungen gegen ein Entgelt anbieten. Ihr Personal (sog. contractors) besteht aus ehemaligen Militärangehörigen, die mit Arbeits- oder Dienstverträgen angestellt sind. Sie werden meist mit einem Tagessatz entlohnt, wobei spezialisierte contractors etwa 20.000 USD im Monat verdienen können. ${ }^{1}$ Kund:innen sind Privatpersonen, Unternehmen, internationale Organisationen, ${ }^{2}$ NGOs und Staaten. Zu den angebotenen Dienstleistungen gehören Transport und Logistik, Versorgung und Verpflegung, Ausbildung und Anleitung, Sicherung von Personen und Objekten sowie Kampfdienstleistungen. ${ }^{3}$

Die Branche wächst zunehmend und hat einen hohen wirtschaftlichen impact factor. So haben die USA von 2009-2018 im Großraum Irak/Afghanistan 208,063 Mrd. USD durch Verträge mit PMCs umgesetzt. Aktuelle Schätzungen gehen von einem Wachstum der Branche auf 420 Mrd. USD innerhalb der nächsten 8 Jahre aus. ${ }^{4}$ Während der Hochphase des Irakkriegs waren genauso viele contractors wie Soldat:innen im Irak durch die USA beschäftigt, im Rahmen der Operation Enduring Freedom übertraf die

1 Salzman, N.Y.U. J. Int'l L. \& Pol. 2008, 853 (885).

2 Crowe/John, Melb. J. Int'l L. 2017, 16 (44).

3 Petersohn, Die Nutzung privater Militärfirmen durch US-Streitkräfte und Bundeswehr, SWP-Studie, S 36, 2006, S. 7; Schreier/Caparini, Privatising Security: Law, Practice and Governance of Private Military and Security Companies, DCAF OC No. 6, 2005, S. 23; Avant, Foreign Policy in Focus 2002, No. 6; Singer, Corporate Warriors 2003, S. 126; Mini, An Analysis of Private Military and Security Companies, EUI Working Paper, AEL 2010/07, 4 f.; Avant/Sigelman, Security Studies 2010, 230 (234f.).

4 Visiongain, Private Military Security Services market set to grow to $\$ 420$ bn by 2029” says new Visiongain report, https://www.visiongain.com/private-militarysecurity- services-market-set-to-grow-to-420bn-by-2029-says-new-visiongain-report/ (letzter Aufruf am 1.4.2021). 
Zahl der contractors die der Soldat:innen zeitweise sogar um etwa 30.000. ${ }^{5}$ Aktuell befinden sich in der Region im Auftrag der USA noch 38.164 contractors. ${ }^{6}$ In der jüngeren Vergangenheit wurde von PMCs in den Konflikten in Donbass, ${ }^{7}$ Jemen, ${ }^{8}$ Syrien, ${ }^{9}$ Bergkarabach,${ }^{10}$ Ukraine, ${ }^{11}$ und der Zentralafrikanischen Republik ${ }^{12}$ berichtet. Der größte Entsendestaat sind nach wie vor die USA, ${ }^{13}$ zunehmend setzen aber auch Russland, ${ }^{14}$ die Türkei und China PMCs ein. ${ }^{15}$

\section{Staatliche Motive für den Einsatz und gegen eine Regulierung von PMCs}

Sparbestrebungen und strategische Motive lassen Staaten PMCs nutzen, obwohl sie auf ein eigenes Militär zurückgreifen können. Aus ähnlichen Gründen verzichten Staaten darauf, nationales oder internationales Recht zur Begrenzung von PMCs zu erschaffen.

5 Peters/Plagakis, Department of Defense Contractor and Troop Levels in Afghanistan and Iraq: 2007 - 2018, Congressional Research Service R44116, S. 6-10.

6 Office of the Assistant Secretary of Defense for Logistics \& Materiel Readiness, CENTCOM|Quarterly Contractor Census Reports, January 2021.

7 Sukhankin, War, Business and Ideology: How Russian Private Military Contractors Pursue Moscow's Interests, https://jamestown.org/program/war-business-andideology-how-russian-private-military-contractors-pursue-moscows-interests/ (letzter Aufruf am 1.4.2021).

8 Hager/Mazzetti, Emirates Secretly Sends Colombian Mercenaries to Yemen Fight, NYT v. 26.11.15, S. A/1.

9 Linder, New Perspectives in Foreign Policy 2018, S. 17; Beslin/Ignjatijevic, Balkan foreign fighters: from Syria to Ukraine, EUISS 2017, 1 (3).

10 Cookman, Syrians Make Up Turkey's Proxy Army in Nagorno-Karabakh, Foreign Policy, https:/foreignpolicy.com/2020/10/05/nagorno-karabakh-syrians-turkey-armenia-azerbaijan/ (letzter Aufruf am 1.4.2021).

11 Reynolds, Putin's Not-So-Secret Mercenaries, Carnegie Endowment for International Peace 2019, S. 2.

12 CAR: Experts alarmed by government's use of "Russian trainers", close contacts with UN peacekeepers https:/www.ohchr.org/EN/NewsEvents/Pages/DisplayNews.aspx?NewsID=26961\&LangID=E (letzter Aufruf am 1.4.2021).

13 Singer, Corporate Warriors (Fn. 3), S. 3.

14 Borshchevskaya, The Role of Russian Private Military Contractors in Africa, Foreign Policy Research Institute, https:/www.fpri.org/article/2020/08/the-roleof-russian-private-military-contractors-in-africa/ (letzter Aufruf am 1.4.2021).

15 Spearin, PRISM 2020, 40 (40-45). 
Der Aufschwung von PMCs fällt in die Zeit der neoliberalen Politik des „schlanken Staates“. ${ }^{16}$ Militärbudgets wurden durch das Ende des Kalten Krieges weltweit verkleinert ${ }^{17}$ und der Markt mit ehemaligen Soldat:innen und Waffen gesättigt. ${ }^{18}$ Contractors sind bereits ausgebildet und müssen nur dann bezahlt werden, wenn sie tatsächlich im Einsatz sind. So entfallen Ausbildungs- und Pensionskosten. Die Spezialisierung der PMCs spricht zudem für schnellere, und dadurch günstigere Einsätze. ${ }^{19}$ Außerdem erlaubt es die privatwirtschaftliche Organisation, die Truppenstärke schneller an konkrete Bedürfnisse anzupassen. ${ }^{20}$ Weitere Einsparungen ergeben sich durch Wettbewerb der PMCs am freien Markt. ${ }^{21}$

Da PMCs gerade keinen Teil der Streitkräfte darstellen, gelten andere formelle Anforderungen an ihre Entsendung. Durch ihren Einsatz kann Außenpolitik durch Exekutivorgane und vorbei an Parlamenten oder internationalen Gremien durchgesetzt werden, sog. foreign policy by proxy. ${ }^{22}$ Außerdem können Einsätze von PMCs ohne den gleichen Rechtfertigungsdruck gegenüber der Zivilgesellschaft durchgeführt werden, da nicht die „eigenen“ Soldat:innen kämpfen. ${ }^{23}$ Prägnante militärische Erfahrungen wie das Somalia-Debakel $1993^{24}$ oder der Vietnamkrieg machen PMCs als Antwort auf neue asymmetrische Kriegsführung auf fremdem Territorium attraktiv. ${ }^{25}$ Teilweise fehlen auch staatliche Truppen, sodass auf Private zurückgegriffen werden muss, um Einsätze durchzuführen. ${ }^{26}$ Die strategischen Motive haben ihren Ursprung im Unterschied zwischen

16 Ettinger, International Journal 2011, 743 (743-764).

17 Leander, Eroding State Authority? Private Military Companies and the Legitimate Use of Force, 2006, S. 33; Krabmann, States, Citizens and the Privatization of Security, 2010, S. 9.

18 Axelrod, Mercenaries, 2013, S. 54.

19 Avant, Orbis 2006, 327 (331 f.).

20 Deitelhoff/Geis, Die Privatisierung des Militärs, in: Leonhard/Werkner (Hrsg.), Militärsoziologie, 2. Aufl., 2012, S. 139 (142).

21 Akcinaroglu/Radziszewski, J. Confl. Resolut. 2013, 795 (803).

22 Michaels, Wash. U. L. Q 2004, 1001 (1062 ff.).

23 Taussig-Rubbo, Yale J.L. \& Human. 2009, 103 (123).

24 Der Schlacht von Mogadischu am 3./4.10.1993 fielen hunderte von Somalis und 19 US-amerikanische Soldaten zum Opfer. Die gescheiterte Operation Gothic Servant wurde 2011 unter dem Titel „Black Hawk Down“ von Ridley Scott verfilmt.

25 Bowden, The Legacy of Black Hawk Down, Smithonian Magazine 2019, https:// www.smithsonianmag.com/history/legacy-black-hawk-down-180971000/ (letzter Aufruf am 1.4.2021).

26 Tepperman, Out of Service, https://newrepublic.com/article/66594/out-service (letzter Aufruf am 1.4.2021). 
Soldat:innen und contractors. Je größer dieser Unterschied in rechtlicher, politischer und symbolischer Hinsicht ist, desto mehr spricht aus staatlicher Sicht für den Einsatz von PMCs.

Die Motive für den Einsatz von PMCs spiegeln sich in den Gründen für die mangelnde Regulierung. Durch eine harte Regulierung würden Kosten entstehen, die die Unternehmen einpreisen würden, denn wirksame Aufsicht und Kontrolle sind teuer. Zudem würde eine Regulierung die Entsendung an formelle Prozesse binden und damit eines der strategischen Hauptmotive für den Einsatz entfallen lassen. ${ }^{27}$ Durch unterschiedliche Erfahrungen der einzelnen Staaten mit PMCs und Söldner:innen gestaltet sich zudem eine internationale Konsensfindung schwierig, da sich Unstimmigkeiten über Inhalt und Form, aber auch die grundsätzliche Frage, ob reguliert werden soll, ergeben.

Insgesamt wollen PMC-einsetzende Staaten keine harte Regulierung, da nur durch weiche Regulierungsformen die Flexibilität des Einsatzes von PMCs beibehalten werden kann. Je regulierter PMCs wären, desto eher würde ihr Einsatz dem von offiziellen Streitkräften gleichen - was ihre Vorteile zunichte machen würde.

\section{Rechtliche Einrabmung}

PMCs agieren nicht in einem rechtsfreien Raum. Allerdings besteht keine Spezialregulierung, die sich direkt mit den Umständen des unternehmerisch organisierten Angebots von Kampfdienstleistungen befasst. Eine von der UN eingesetzte Working Group wurde mit der Ausarbeitung eines bindenden internationalen Vertrages zur Regulierung von PMCs betraut, scheiterte aber an Unstimmigkeiten über die Rechtsqualität dieses Vertrags. Einer nachfolgenden Working Group wurde sodann das Mandat für einen bindenden Vertragstext entzogen. ${ }^{28}$ Auf internationaler Ebene besteht weiterhin keine Spezialregulierung.

Aber auch ohne speziell auf sie zugeschnittene Normen müssen sich PMCs bestehenden und auf sie anwendbaren Regelungen unterwerfen, die ihre Handlungsspielräume begrenzen und Rechtsschutz für Betroffene bieten könnten. In Betracht kommen das humanitäre Völkerrecht, Grundsät-

27 Lehnardt, Private Military Companies and State Responsibility, IILJ Working Paper 2007/2, S. 2.

$28 \mathrm{UN} / \mathrm{A} / \mathrm{HRC} / \mathrm{Res} / 36 / 11$. 
ze der Staatenverantwortlichkeit, Völkerstrafrecht, sowie nationalstaatliche Regulierungen.

\section{Humanitäres Völkerrecht}

Contractors agieren in international bewaffneten Konflikten und üben potentiell konfliktbezogene Gewalt aus, sie bewegen sich damit grundsätzlich im Anwendungsbereich des humanitären Völkerrechts.

Das humanitäre Völkerrecht teilt die vom Konflikt Betroffenen in Kombattant:innen und Zivilist:innen ein. Das Verhalten von Kombattant:innen ist in bewaffneten Konflikten den einsetzenden Staaten zurechenbar, ${ }^{29}$ zudem gilt eine individuelle Verantwortlichkeit für humanitärem Völkerrecht entgegenstehendes Verhalten. Kombattant:innen sind der normative Grundfall von Personen, die Schädigungshandlungen im Konflikt ausüben und werden daher engmaschig reguliert und kontrolliert. Wären contractors auch Kombattant:innen, würden sie dementsprechend haften, etablierte Kontrollelemente könnten angewendet werden.

Allerdings mangelt es privaten Dienstleistenden an einem geforderten engen Verbindungsband zum einsetzenden Staat, um eine Kombattant:inneneigenschaft nach Art. 4(A) der Dritten Genfer Konvention (GK III) begründen zu können. Da contractors weder formell in die Streitkräfte eingegliedert sind, ${ }^{30}$ noch die vom humanitären Völkerrecht geforderte verantwortliche Führung eines Freiwilligencorps vorweisen können, ${ }^{31}$ oder mangels Friedlichkeit als Personen, die den bewaffneten Personen folgen, zu qualifizieren sind, ${ }^{32}$ vermag keine der Normvarianten zum Kombattant:innenstatus zu verhelfen. ${ }^{33}$

Wer nicht Kombattant:in ist, kann nur Zivilist:in sein, das gilt auch für contractors. ${ }^{34}$ Wenn sie an Konflikthandlungen teilnehmen, verlieren sie für diese Zeit ihren Schutzstatus, Art. 51 Nr. 3 des Ersten Zusatzprotokolls

29 ICRC, Study on customary international humanitarian law, 2005, Rule 149.

30 Lehnardt, Private Militärfirmen und völkerrechtliche Verantwortlichkeit, 2011, S. $141 \mathrm{f}$.

31 Zum Teil mit anderem Ergebnis: Fischer, Militär- und Sicherheitsunternehmen in bewaffneten Konflikten und Friedenssicherungsoperationen, 2013, S. 94 f.

32 Gillard, in: Alexandra/Baker/Caparini (Hrsg.), Private Military and Security Companies, 2008, S. 159 (163 f.).

33 Gleiches gilt für Art. 43 ZP I, der ähnliche Voraussetzungen aufstellt, siehe Schüller, Sicherheit und Frieden 2008, 191 (192).

34 Das legen z.B. die USA für PMCs fest, die die staatlichen Truppen begleiten: Code of Federal Regulation, 48 CFR $\$ 52.225-19$, (b)(3). 
(ZPI). Ausgeübte Schädigungshandlungen unterfallen dem allgemeinen Strafrecht, wobei die Teilnahme an Feindseligkeiten an sich keine Straftat darstellt. ${ }^{35}$ Spezielle Rechte und Pflichten im bewaffneten Konflikt bestehen aber nicht.

Einen Sonderstatus im humanitären Völkerrecht haben Söldner:innen nach Art. 47 ZPI inne. Sie sind weder privilegiert noch geschützt und erhalten auch keinen Kriegsgefangenenstatus. Verschiedene internationale Konventionen verbieten ihren Einsatz und ihre Unterstützung. ${ }^{36}$ Als Söldner:innen könnten contractors für Handlungen und für ihr Dasein als Söldner:innen strafrechtlich verfolgt werden. Dabei gelten die Garantien aus Art. 75 ZPI. ${ }^{37}$

Auf den ersten Blick scheinen contractors den normativen Vorstellungen von Söldern:innen zu entsprechen, Presse und (Populär-)Wissenschaft bezeichnen sie auch oft so. ${ }^{38}$ Allerdings ist die Söldner:innendefinition so mangelhaft konzipiert, dass sie kaum Anwendung findet. ${ }^{39}$ Durch ein Festhalten an sechs kumulativ zu erfüllenden Voraussetzungen, insbesondere einem negativen Staatsangehörigkeitserfordernis und Gewinnstreben als Hauptmotiv, wird der Tatbestand so verengt, ${ }^{40}$ dass nicht nur contractors keine Söldner:innen darstellen. ${ }^{41}$ Völkerrechtlich gibt es, seitdem die normative Ächtung des Söldnertums ins Recht transkribiert wurde, faktisch keine Söldner:innen mehr.

Es gilt daher kein spezieller Regulierungsrahmen oder ein Verbot für PMCs im humanitären Völkerrecht.

35 Gasser/Dörmann, The Protection of the Civilian Population, in: Fleck (Hrsg.), Handbook of International Humanitarian Law, 3. Aufl., 2013, Rn. 517/2.

36 Organisation of African Unity, Convention for the Elimination of Mercenarism in Africa, 1977, Art. 1; UN, International Convention against the Recruitment, Use, Financing and Training of Mercenaries, 2001, Art. 1; ILC Draft Code of Crimes against the Peace and Security of Mankind, 1991, Art. 23(2).

$37 \operatorname{ICRC~(Fn.~29),~Rule~} 108$.

38 Hager/Mazzetti (Fn. 8); Security Council Report, April 2021, https://www.securitycouncilreport.org/monthly-forecast/2021-04/in-hindsight-guns-for-hire-the-security-council-and-mercenarism.php (letzter Aufruf am 1.4.2021).

39 Hampson, Netherlands Yearbook of International Law 22 (1991), 3 (30).

40 Percy, Mercenaries, 2007, S. 177.

41 UN/A/HRC/WG.10/2/CRP.1, S. 6. 


\section{Staatenverantwortlichkeit}

Der Grundsatz der Staatenverantwortlichkeit unterscheidet zwischen Handlungen Privater und des Staates. Nur für letztere sind Staaten grundsätzlich verantwortlich. Mangels formeller Inkorporation in die Streitkräfte sind Angestellte von PMCs Private. Eine Staatenverantwortlichkeit kann sich daher nur ergeben, wenn entweder das Handeln der PMCs dem Staat zugerechnet werden kann oder eine due diligence Verletzung des Staates vorliegt.

Eine Zurechnung kann stattfinden, wenn ein Staat Dienstleistende autorisiert, staatliche Gewalt auszuüben, Art. 5 International Law Commission, Articles on State Responsibility (ILC). ${ }^{42}$ Was genau unter Staatsgewalt zu verstehen ist, wird im internationalen Recht nicht definiert. Die USA fassen darunter z.B. die Handlungen, die so eng mit dem öffentlichen Interesse verbunden sind, dass sie nur von Staatsbediensteten ausgeübt werden dürfen. ${ }^{43}$ Hier beißt sich die Katze in den Schwanz: Wenn PMCs handeln, findet ja gerade eine Auslagerung statt. ${ }^{44}$ Es kann sich dann nicht um Staatsgewalt handeln. ${ }^{45}$ Mehrdeutige Vertragsbestimmungen werden zudem bewusst eingesetzt, um zu verschleiern, welche Tätigkeiten durch PMCs genau ausgeübt werden. ${ }^{46}$ Staatenverantwortlichkeit über eine Zurechnung privaten Verhaltens ist daher nur in sehr begrenzten Einzelfällen vorstellbar, etwa wenn contractors als Gefängniswärter:innen agieren und gerichtlich angeordnete Disziplinarmaßnahmen durchsetzen. ${ }^{47}$

Jenseits einer ausdrücklichen Autorisierung kann eine Zurechnung erfolgen, wenn PMCs unter staatlicher Anleitung, Führung oder Kontrolle handeln, Art. 8 ILC. Abgesehen von direkten Anweisungen zu Menschenrechtsverletzungen ist unklar, wie eng die Einwirkung auf die Privaten sein muss. Der Internationale Gerichtshof geht davon aus, dass es „effektiver Kontrolle" bedarf, mithin spezifischer Instruktionen zur Ausführung einer bestimmten Tätigkeit. Contractors fallen nicht unter militärische Kommandostrukturen, das Verhältnis von PMCs und Militär ist vielmehr

42 UN/A/56/49(Vol. I)/Corr.4.

43 Federal Activities Inventory Reform Act of 1998, Public Law 105-270 and Federal Acquisition Regulation of 2005; Office of Management and Budget, Circular A-76 (revised), 29 May 2003, A-2.

44 Im Irak übten PMCs „,inherently governmental functions“ und ,inherently soldierly“ functions aus, siehe Michaels, Wash. U. L. Q 2004, 1001 (1031).

45 Lehnardt, State Responsibility (Fn. 27), S. 8.

46 Ebd., S. 10.

47 ILC Commentary to the Draft Articles on State Responsibility, Art. 5 (2). 
als „informelle Koordination“ zu beschreiben. Inwieweit direkte Anordnungen für konkrete Handlungen i. S. e. effektiven Kontrolle erteilt und umgesetzt werden, ist unklar. Die bestehende Abhängigkeit von Finanzierung reicht z.B. nicht aus. ${ }^{48}$

Im Rahmen ihrer menschenrechtlichen Verantwortung von respect, protect, fulfil ${ }^{49}$ haben Staaten die Verpflichtung zur Gewährleistung von Menschenrechten und müssen ggf. auch gegen Dritte vorgehen, wenn diese Menschenrechte verletzen. Die Verletzung liegt dann nicht in der Handlung der Privaten, sondern im staatlichen Unterlassen, eine sog. due diligence Verletzung. Welche Sorgfalt Staaten aber im Rahmen dieser Verpflichtung schulden, ist hoch umstritten. ${ }^{50}$ Ob eine unsichere Staatenverantwortlichkeit eine individuelle Haftung ausgleichen oder Staaten von einem Anwerben von PMCs abhalten kann, ist mehr als fraglich. Eine Staatenverantwortlichkeit für Handlungen von PMCs oder im Rahmen einer due diligence Verpflichtung wurde zudem bisher noch nicht ausgelöst ${ }^{51}$ oder geltend gemacht. ${ }^{52}$

\section{Völkerstrafrecht}

Um nicht-staatliche Individuen wie contractors durch das Römische Statut zu erfassen, müssen diese eine Verbindung zum kriegsführenden Staat aufweisen. ${ }^{53}$ Wie eng diese sein muss, ist umstritten. ${ }^{54}$ Würden ähnlich strikte Verbindungen wie bei der Staatenverantwortlichkeit gefordert, bestünden zumindest die bereits angesprochenen Probleme um die Ausübung von Staatsgewalt.

48 ICJ, Case concerning Military and Paramilitary Activities in and against Nicaragua (Nicaragua v. United States), Judgment (Merits), 27.6.1986, ICJ Reports 1986, Rz. $188 \mathrm{f}$.

49 UN/CCPR/C/21/Rev.1/Add. 13; Karp, International Theory (2020), 83 (83-108); De Schutter, International Human Rights Law (2010), S. 416-512.

50 Garcia Amador, Second Report on State Responsibility, YILC 1957 II, S. 104.

51 UN/A/HRC/WG.10/2/CRP.1, S. 7.

52 Lehnardt, State Responsibility (Fn. 27), S. 19.

53 ICTR Trial Chamber, Prosecutor v. Akayesu, ICTR-96-4, judgment, 2.9.1998, Rz. 631; ICTR Trial Chamber, Prosecutor v. Kayishema and Ruzindana, ICTR-95-1, judgment, 21.5.1999, Rz. 174-176.

54 Lehnardt, EJIL 2008, 1015 (1019). 
Hinzu kommt, dass die zu ahnende Tat im Zusammenhang mit dem bewaffneten Konflikt stehen muss. ${ }^{55}$ Der bewaffnete Konflikt muss ein entscheidender Faktor für die Möglichkeit und die Art der Begehung der Tat gespielt haben. ${ }^{56}$ Diese Verbindung ergibt sich z.B. durch die Kombattant:inneneigenschaft, die Zugehörigkeit zu einer Konfliktpartei oder die Begehung der Tat in Ausübung einer offiziellen Verpflichtung oder zur Unterstützung eines militärischen Ziels. ${ }^{57}$ Zumindest Kombattant:innen sind contractors, wie aufgezeigt, nicht. Entscheidend ist daher wieder die Nähe des/der Täter:in zum Konflikt.

Dieses geforderte doppelte Näheverhältnis wird nur für wenige Angestellte von PMCs zu bejahen sein. Erfasst werden könnten z.B. Sicherheitsdienstleistende für Diplomat:innen oder Gefägniswärter:innen. Aber selbst für diese eng gebundenen PMCs sind bisher keine Prozesse angestrebt worden. So waren an der Folter in Abu Ghraib auch contractors beteiligt, die jedoch nicht zur Rechenschaft gezogen wurden. ${ }^{58}$ Die Nichtanwendung von Völkerstrafrecht gegen contractors zeigt, dass die Durchsetzung handlungsbegrenzender Normen gegenüber contractors keine Priorität der internationalen Gemeinschaft darstellt. ${ }^{59}$

\section{Nationalstaatliche Regulierung}

Militär- und Polizeiaufgaben, die Tätigkeitsfelder, die zumeist an PMCs ausgelagert werden, sind hochgradig regulierte Bereiche. Es fehlt jedoch an einer auch nur ansatzweise vergleichbaren Regulierung von PMCs. ${ }^{60}$ Viele Staaten haben keine spezifisch auf PMCs zugeschnittene Gesetze,

55 ICTY, Prosecutor v. Ljube Boškoski and Johan Tarčulovski, ICTY-IT-04-82-T, 10.7.2008, Rz. 239; ICC Pre Trail Chamber, Prosecutor v. Germain Katanga and Mathieu Ngudjolo Chui, ICC-01/04-01/07, 30.9.2008, Rz. 382.

56 Dörmann, Max Planck Yearbook on of United Nations Vol. 7 (2003), 341 (358).

57 ICTY Appeals Chamber, Prosecutor v. Zejnil Delialic, Zdravko Mucić (aka "PAVO”), Hazim Delic and Esad Landžo (aka “ZENGA”), IT-96-21-, 20.2.2001, Rz. 325; Stella Ageli, Amsterdam Law Forum 2016, 28 (40 ff.).

58 Fay, AR 15-6 Investigation of the Abu Ghraib Detention Facility and 205th Military Intelligence Brigade 68-95 2004, S. 79, 136; Spiegel, No contractors yet face Abu Ghraib charges, Financial Times, https:/www.ft.com/content/ a5d112a4-082a-11da-97a6-00000e2511c8 (letzter Aufruf am 1.4.2021).

59 Lehnardt, EJIL 2008, 1015 (1034).

60 UN/A/HRC/WG.10/2/CRP.1, S. 10. 
wie etwa der überwiegende Teil der europäischen Staaten. ${ }^{61}$ Wenn gesonderte Bestimmungen bestehen, behandeln diese vornehmlich die Aufsicht von Sicherheitsgewerben im Inland und die damit verbundene Ein- und Ausfuhr von Waffen, ${ }^{62}$ wie z.B. in Großbritannien, ${ }^{63}$ Ungarn $^{64}$ und Frankreich. ${ }^{65}$ Eine Ausnahme stellt die Schweiz dar, die auch kampfbezogene Handlungen im Ausland erfasst. ${ }^{66}$

Ein anderes Bild zeichnet sich für die Staaten in Zentral- und Südamerika sowie Asien. ${ }^{67}$ Hier bestehen oft nationalstaatliche Spezialgesetze über PMCs. Das kann an anderen Erfahrungen mit Söldern:innen und privaten Militärunternehmen liegen, die diese Länder z.B. im Rahmen der Dekolonisation machen mussten. Jedoch wird auch hier zumeist nur der Einsatz im Inneren und nicht die Teilnahme an Feindseligkeiten im Ausland geregelt. ${ }^{68}$ Selten wird ein Verbot von militärnahen Dienstleistungen ausgesprochen, wie etwa in Russland, welches erstaunlicherweise gleichwohl PMCs einsetzt. ${ }^{69}$ In Südafrika unterliegen Dienstleistungen von PMCs einem Erlaubnisvorbehalt. ${ }^{70}$

Die USA regeln auf Bundesebene die Privatisierung von öffentlichen Aufgaben nur im Allgemeinen. Unterschiedliche Anweisungen und Verordnungen des Department of Defense und Militärhandbücher beziehen sich hingegen konkret auf PMCs. Zusammen ergibt sich eine Regulierungslage, die die Privatisierung von inhärent staatlichen Funktionen verbietet, gleichwohl aber die Durchführung einer Zusammenarbeit mit PMCs auch in solchen Situationen umschreibt. Es mangelt an Regelungen

61 https://www.ohchr.org/Documents/Issues/Mercenaries/WG/Legislation/EuropeSt udy.doc (letzter Aufruf am 1.4.2021).

62 Working Group on mercenaries, Mercenarism and Private Military and Security Companies (2018), S. 19.

63 Private Security Industry Act 2001.

64 Act CXXXIII of 2005 on the rules of personal and property protection activities and private investigation; Act $\mathrm{C}$ of 2012 on the Criminal Code.

65 Loi $\mathrm{n}^{\circ}$ 83-629 du 12.7.1983 réglementant les activités privées de surveillance, de gardiennage et de transport de fonds; Décret $n^{\circ}$ 2005-1122 du 6.9.2005; Décret $n^{\circ}$ 2009-137 du 9.2.2009; Décret n²009-214 du 23.2.2009.

66 Loi fédérale sur les prestations de sécurité privées fournis à l'étranger du 27 septembre 2013.

67 UN/A/HRC/36/47, S. 3-15.

68 A/HRC/27/50, S. 6; UN/A/HRC/24/45, S. 6-7.

69 Ostensen/Bukkvoll, Russian Use of Private Military and Security Companies - the implications for European and Norwegian Security, FFI-Report 18/01300, S. 3, 13; Eklund/Elving, Eurasia Daily Monitor Vol. 14, Issue 39.

70 Prohibition of Mercenary Activities and Regulation of Certain Activities in Country of Armed Conflict, Act No. 27 of 2006. 
über die andauernde Kontrolle von Unternehmen und zur Durchsetzung von Strafansprüchen. Die Bestimmungen behandeln vermehrt Aspekte der Zusammenarbeit, arbeitsschutzrechtliche Inhalte, oder Verfügungen über das Tragen von Waffen und Uniformen. ${ }^{71}$ Gewalt soll von contractors nur dann angewendet werden, wenn sie vernünftigerweise notwendig ist, um die konkreten Aufgaben ausführen zu können, ${ }^{72}$ stellenweise wird auf eine rein defensive Gewaltanwendung hingewiesen. Die Vorgaben sind teilweise widersprüchlich und werden in der Praxis nicht angewendet: Offensivaktionen von PMCs sind bekannt und werden angefordert. ${ }^{73}$ Die dünne und unstrukturierte Regulierungslage kann ihrer mangelnden Substanz nicht mit Durchsetzungskraft entgegentreten: Verurteilungen von contractors sind fast nicht anzutreffen. Außer im Falle von vier Angestellten der PMC Blackwater (jetzt Academi), die für das tödliche Massaker auf dem Nisourplatz verantwortlich waren, sind kaum Verurteilungen bekannt (die vier Verurteilten wurden zudem im Dezember 2020 begnadigt, was massive internationale Kritik auslöste). ${ }^{74}$ Selbst wenn contractors in den USA zivilgerichtlich zur Verantwortung gezogen werden sollten, scheiterten die Verfahren an der Geltendmachung von Verfahrenshindernissen wie dem Verrat von Staatsgeheimnissen ${ }^{75}$ oder der Berufung auf Immunitäten, wie sie staatliche Militärangehörige geltend machen können („,combat immunity" und „battlefield preemption“). ${ }^{76}$

Die Staaten, in denen PMCs eingesetzt werden, haben durch ihre territoriale Hoheit grundsätzlich die Aufgabe der Strafverfolgung und können auch zivilrechtlich regulieren. Allerdings sind diese Staaten gerade durch einen bewaffneten Konflikt institutionell und strukturell geschwächt; Strafverfolgung und auch Beweissicherung finden, wenn überhaupt, unter ungünstigen Bedingungen statt. ${ }^{77}$ Erschwerend tritt hinzu, dass Entsende-

71 Siehe z.B. Code of Federal Regulations, 48 CFR $\$ 252.225-7040$.

72 Department of Defense Directive No. 5210.56 of 1 April 2011, enclosure 2, section 2 (a).

73 Siehe z.B. für den Irak: Michaels, Wash. U. L. Q 2004, 1001 (1033-1034).

74 US pardons for Blackwater guards an "affront to justice", https://www.ohchr.org/en/NewsEvents/Pages/DisplayNews.aspx?NewsID=26633\&LangID=E (letzter Aufruf am 1.4.2021); Falih Hassan/Jane Arraf, Blackwater's Bullets Scarred Iraqis. Trump's Pardon Renewed the Pain, NYT v. 24.12.2020, S. A/1.

75 Mohamed v. Jeppesen Dataplan, 614 F.3d 1070 (9th Cir. 2010).

76 Saleh v. Titan Corp., 580 F.3d 1 (D.C. Cir. 2009); Al-quraishi v. L-3 Serv., 657 F.3d 201 (4th Cir. 2011); Al Shimari v. Caci International, Inc., 658 F.3d 413 (4th Cir. 2011).

77 UN/A/HRC/WG.10/2/CRP.1, S. 10-11. 
staaten oft mit Aufnahmestaaten internationale Verträge abschließen, die eine Strafverfolgung von PMCs durch den Aufnahmestaat unterbinden, ${ }^{78}$ was aber wiederum nicht von einer engeren Aufsicht durch den Entsendestaat aufgefangen wird.

Auch nationalstaatliche Regulierung von unterschiedlichen Zugriffspunkten heraus kann kein engmaschiges Netz erzeugen, welches zuverlässig zu einer Verantwortlichkeit von PMCs für schädigendes Verhalten führt. Strafrechtliche Sanktionierungen oder durchsetzbare Rechtsbehelfe für Opfer fehlen. ${ }^{79}$ Durch die Regulierungslücken ist nicht nur das Recht auf Leben und körperliche Unversehrtheit, oder die Verbote von Freiheitsentzug und Folter beeinträchtigt, auch soziale und kulturelle Rechte sowie das Recht der Selbstbestimmung der Völker können durch den unregulierten Einsatz von PMCs bedroht sein. ${ }^{80}$

Auf internationaler Ebene erfasst das veraltete humanitäre Völkerrecht contractors nicht, auch die Grundsätze der Staatenverantwortlichkeit und das Völkerstrafrecht können eine Haftung für PMCs und ihre Angestellten nicht klar absichern. Die unzulängliche Regulierungslage wird durch ein Nichtinteresse an Verfolgung ergänzt. ${ }^{81}$ Es besteht mithin tatsächlich eine gravierende Regulierungs- und Haftungslücke für PMCs, die Gewalt in bewaffneten Konflikten ausüben.

\section{Selbstregulierung}

In diese regulierungsarme Lage, in dieses „rechtliche Vakuum“, 82 tritt Selbstregulierung ein. Selbstregulierung ist ein regulatorischer Prozess, der auf Industrieebene vorgenommen wird und Regeln und Standards für eben diese Industrie setzt. ${ }^{83} \mathrm{Im}$ Gegensatz zu staatlicher Regulierung auf Gesetzesbasis, aus der Rechte und Pflichten folgen, stellt Selbstregulierung eine freiwillige Selbstverpflichtung, mithin soft law dar. ${ }^{84}$ Selbstregu-

78 Siehe z.B. die gesetzliche Verankerung in 48 CFR $\$ 252.225-7040$, (b)(4).

79 Working Group on mercenaries (Fn. 62), S. 18.

80 Ebd.; International Federation for Human Rights, Open Letter: Supporting the Cambodian Land Grabbing Case, https:/www.fidh.org/IMG/pdf/cambodiaopen_letter_to_icc_pros-16_march_2021.docx.pdf (letzter Aufruf am 1.4.2021).

81 Jung, Harvard International Review 2016, 7 (8).

82 Singer, Colum. J. Transnat'l L. 2004, 521, (524, 541).

83 Gunningham/Rees, Law \& Policy 1997, 363 (364f.).

84 DeWinter-Schmitt, in: Torroja (Hrsg.), Public International Law and Human Rights Violations by Private Military and Security Companies, 2017, 105 (105 ff.). 
lierung wird hauptsächlich für zwei Zwecke eingesetzt: Staatliche Regulierung soll abgewehrt und Reputationspflege betrieben werden. ${ }^{85} \mathrm{Um}$ ein Eingreifen durch staatliche Regulierung zu verhindern, muss das eigene System funktionieren. Mittel dafür sind Überwachung und Sanktionen. Daneben wird die Selbstregulierung mit anderen Vorteilen verknüpft, um die Anzahl der Teilnehmenden zu erhöhen. ${ }^{86}$ Passend titelt die International Code of Conduct Association: „Doing the right thing makes better business sense ". ${ }^{87}$ Der Inhalt der Selbstregulierung ist doppelt ausschlaggebend für deren Erfolg: Einerseits muss der Inhalt „erfüllbar" sein, ohne die Handlungsspielräume der PMCs zu sehr einzuschränken, damit viele Akteure mitmachen. Andererseits muss der Inhalt so signifikant sein, dass die Selbstverpflichtung als wirksam gelten kann und staatliche Regulierung weiter abgehalten wird.

Für PMCs bestehen viele unterschiedliche Selbstregulierungsregime. ${ }^{88}$ Zwei prominente Mechanismen sind das Montreux Document und der International Code of Conduct $(\mathrm{ICoC})$. Während das Montreux Document Staaten anspricht, wendet sich der $I C o C$ direkt an PMCs.

\section{Montreux Document}

Das Montreux-Document wurde auf Initiative der Schweiz und des Internationalen Komitee des Roten Kreuzes von 18 Staaten, Branchenvertreter:innen und NGOs erarbeitet und ist mittlerweile von 57 Staaten, sowie der EU, OSZE und NATO gezeichnet. ${ }^{89}$ Trotz der Mitwirkung und Unterzeichnung von Staaten ist es kein internationaler Vertrag. Die Selbstbezeichnung lautet ,intergouvernementales Dokument“ ${ }^{\circ 0}$ Es werden keine neuen Normen erschaffen, weder Staaten noch PMCs werden Verpflichtungen auferlegt. Vielmehr gibt das Dokument diejenigen Regelungen aus dem Völkerrecht und dem Recht der Staatenverantwortlichkeit wieder, die

85 Héritier/Eckert, Journal of Public Policy 2008, 113 (116ff.); Mattli/Buthe, World Politics 2003, 1 (18 ff.); Jägers, Human Rights \& International Legal Discourse 2012, 56 (68).

86 De Nevers, Journal of Public Policy 2010, 219 (224).

87 ICoCA, https://icoca.ch/private-security-companies/ (letzter Aufruf am 1.4.2021).

88 Quirico, J. TRANSNAT'L L. \& POL'Y. 2017/2018, 67 (72).

89 https://www.eda.admin.ch/eda/en/home/foreign-policy/international-law/internat ional-humanitarian-law/private-military-security-companies/participating-states.ht $\mathrm{ml}$ (letzter Aufruf am 1.4.2021).

90 https://www.mdforum.ch/pdf/The-Montreux-Document-in-a-Nutshell.pdf (letzter Aufruf am 1.4.2021). 
sowieso für Staaten gelten, die PMCs einsetzen - und die, wie aufgezeigt, nicht zur Anwendung kommen. Es ist daher eine Zusammenstellung von Rechtstexten - etwa wie der Sartorius II. Es hat keinerlei eigenständige Rechtsqualität und bringt keinerlei Neuerungen.

Das Montreux Document stellt mithin nicht einmal eine freiwillige Selbstverpflichtung dar, da keine weitergehenden Verbindlichkeiten übernommen werden, als durch das internationale Recht schon bestehen. Es wird nur der Eindruck erweckt, etwas zur regulatorischen Fülle beizutragen; dabei werden die klassischen Zwecke von Selbstregulierung erfüllt: Durch ein Zugriffsrecht auf die Materie wird der Einfluss von staatlicher, d.h. in diesem Falle rechtsqualitativer Regulierung abgewendet. Gleichzeitig wird Reputationspflege betrieben. Besonders ist, dass hier Staaten das Instrument Selbstregulierung gewählt haben, obwohl ihnen kollektiv wie individuell harte Regulierungsformen zur Verfügung stünden. Sie nutzen bewusst soft law, um dem Eindruck eines regulatorischen Vakuums entgegenzutreten, den Einsatz von PMCs zu legitimieren und in Initiativen für rechtsqualitative Instrumente Gegenargumente vortragen zu können. ${ }^{91}$

\section{International Code of Conduct}

Der ICoC ist der komplementäre Teil zum Montreux Document und richtet sich direkt an PMCs. Die International Code of Conduct Association (ICoCA) ist für die Überwachung der Umsetzung des $I C o C$, die Lizensierung und für die Aufsicht über die partizipierenden Unternehmen zuständig. Die ICoCA besteht aus Mitgliedern, die den drei Säulen Staaten, PMCs und zivilgesellschaftliche Organisationen zugeordnet werden können, Art.3.1 Articles of Association (AoA). Derzeit besteht das Board of Directors aus zwei Staatenvertreter:innen und jeweils vier Repräsentant:innen der PMCBranche und der Zivilgesellschaft.

Der Code will gemeinsame Standards setzen, sowie Aufsicht und Kontrolle etablieren $(\$ 5)$. Gleichzeitig besitzt der $I C o C$ die gleiche Rechtsqualität wie das Montreux Document - nämlich keine - da er ausdrücklich keine eigenen rechtlichen Verpflichtungen oder Verbindlichkeiten kreiert ( $\mathbb{1 4}$ 14. ${ }^{92}$ Dazu passt, dass Unternehmen, die dem Code beitreten, das Mon-

91 Saner, Private Military and Security Companies, CCDP Working Paper (2015), S. 14.

92 "This Code complements and does not replace the control exercised by Competent Authorities, and does not limit or alter applicable international law or relevant national law. The Code itself creates no legal obligations and no legal 
treux Document und die UN Principles on Business and Human Rights ${ }^{93}$ befürworten müssen $(\$ 3)$. Die Anerkennung bestehender international-rechtlicher Verpflichtungen von Staaten durch private Unternehmen stellt aber natürlich nicht mehr als eine nichtssagende Floskel dar.

Einzelne Handlungsanweisungen werden in $\$ 22$ und $\$ \$ 28-43$ umschrieben. Dabei hat sich der Code in der Auswahl der aufgeführten Verbrechen am Römischen Statut orientiert. Auch für die einzelnen Tatbestände gilt, dass keine Konsequenzen mit ihnen verbunden sind: Eine über bestehende Verpflichtungen herausgehende Wirkung kann der Selbstverpflichtung nicht zugesprochen werden, siehe $\$ 14$. Ein echter Mehrwert ergibt sich daher nicht.

Durch Kontrollmechanismen soll die Einhaltung des ICoC überwacht werden. Angedacht sind dafür Dialoge, Selbstauskünfte, media-monitoring und Begehungen vor Ort, Art. 12 AoA. Obwohl die ICoCA und der Code seit 2010 bestehen, sind zur Ausführung benötigte Institutionen und Prozesse noch nicht vollständig aufgebaut. Es besteht auch die Möglichkeit, bei der ICoCA Beschwerden einzureichen. ${ }^{94}$ Seit 2015 wurden 33 Beschwerden bei etwa 900 überwachten Unternehmen erfasst. Bei keiner Beschwerde wurde eine Code-Verletzung festgestellt. Diesen Zahlen sollte mit äußerster Skepsis begegnet werden.

Unklar bleiben auch die Rechtsfolgen eines Verstoßes. Der Code wirkt, neben seiner mangelnden Rechtsqualität, nur inter-partes zwischen dem Unternehmen und dem anstellenden Staat. Welche Wiedergutmachungsmechanismen können eingesetzt werden? Wer ist überhaupt der/die Betroffene? Individuen, die Gewalt erfahren haben oder Vertragspartner:innen? $\mathrm{Ob}$ und wie daraus eine Art Rechtsbehelf für Betroffene werden soll, ist vollkommen unklar. Ob eine Aufarbeitung oder Kompensation nach einer Beschwerde erfolgt, wird nicht konkretisiert. Da es nach eigener Anamnese keine Verstöße gibt, hat die Erarbeitung dieser Prozesse wohl keine Priorität. Weniger als 50\% der Verträge der USA mit PMCs halten überhaupt einen Kontrollmechanismus zur Einhaltung der Vertragsbedingungen (zu denen dann der Code gehören müsste) vor. ${ }^{95}$

liabilities on the Signatory Companies, beyond those which already exist under national or international law. Nothing in this Code shall be interpreted as limiting or prejudicing in any way existing or developing rules of international law.”, $\$ 14$ ICoC.

$93 \mathrm{~A} / \mathrm{HRC} / 17 / 31$.

94 https://icoca.ch/registering-a-complaint/ (letzter Aufruf am 1.4.2021).

95 Isenberg, Private Military Contractors and U.S. Grand Strategy, 2009, S. 23. 
Auch als Grundlage von Verfahren vor staatlichen Gerichten zwischen contractors und Betroffenen eignet sich der Code mangels Rechtsqualität nicht. Der Code ist damit für Betroffene von Schädigungshandlungen nutzlos. Auch präventiv kann der Code so nicht wirken.

Gleichwohl wird die „Zertifizierung" durch den Code von den Unternehmen prominent eingesetzt und soll den Anschein von besonderer Güte erwecken. Reputationspflege wird vollumfänglich betrieben und durch den Code staatliche Rechtssetzung abgewehrt. Besonders wirkungsvoll sind die beiden Zwecke der Selbstregulierung dadurch umgesetzt, dass das soft law durch staatliche Beteiligung am Entstehungsprozess zusätzliche Glaubwürdigkeit und damit Wirkungsmacht erhält.

Das Montreux Document und der International Code of Condunct sind damit Beispiele von Selbstregulierung, die ihre Zwecke aus Unternehmenssicht erfüllen, aber keine Änderung der Rechtslage um PMCs ergeben. ${ }^{96}$

\section{Machtverschiebungen}

Private Militärunternehmen agieren in Kriegs- und Nachkriegsgebieten, wenden Gewalt an und üben dabei zum Teil Staatsgewalt aus. Gleichwohl werden sie von den bestehenden Regelungen kaum erfasst, zumindest werden diese nicht auf sie angewendet. Die Selbstregulierung der Branche ist nicht geeignet, das transnationale Geschäft in einer globalisierten Welt regulatorisch einzufassen oder den Zugang zu staatlichen Gerichten zu ermöglichen. ${ }^{97}$ Das macht den Einsatz von PMCs für Staaten attraktiv, da sie PMCs anders als reguläre Streitkräfte einsetzen können. Gleichzeitig entstehen dadurch Machtverschiebungen zwischen Staaten, PMCs und Betroffenen.

\section{Verhältnis PMC-Betroffene}

Die erste Machtverschiebung ergibt sich im Verhältnis von PMCs und den von ihrer Gewaltausübung Betroffenen zugunsten der Unternehmen. Durch den Rückzug der Staaten aus ihrer gesetzgeberischen Verantwortung und der damit einhergehenden Abhängigkeit von Selbstregulierung

96 Ähnlich verhält es sich mit anderen, weniger prominenten Selbstverpflichtungsinstrumenten, siehe De Nevers, Journal of Public Policy 2010, 219.

97 UN/A/HRC/WG.10/2/CRP.1, S. 10. 
haben PMCs zweifach Einwirkungsmöglichkeiten erlangt. Erstens haben sie die Möglichkeit, das Recht schaffen zu können, welches für sie gelten soll, indem sie Selbstregulierung vornehmen. Zweitens können sie durch das Schaffen von Selbstverpflichtungen auf die Erhaltung des unregulierten status quo Einfluss nehmen. Betroffene von Schädigungshandlungen hingegen sind auf durchsetzbare Normen angewiesen, auf die sie nur sehr mittelbar als Bürger:innen eines Staates Einfluss nehmen können. Obwohl sich zwei Private gegenüberstehen, können die Unternehmen staatsähnliche Macht in der Zweierbeziehung ausüben.

Die Einseitigkeit der Situation wird durch den prekären Zugang zu gerichtlicher Kontrolle verstärkt. Der Handlungsspielraum von PMCs erweitert sich daher durch Selbstregulierung über die (vormals) gesetzlichen Grenzen hinweg, da sie keine individuelle Verantwortlichkeit zu fürchten brauchen. Vulnerable Betroffene von Gewalt sind daher durch Selbstregulierung nicht bessergestellt. Vielmehr findet durch die Verdrängung von staatlicher Regulierung eine Schlechterstellung statt. Irgendeine Regulierung ist (in diesem Fall) nicht besser, als gar keine Regulierung.

\section{Verhältnis Staat-PMC}

Während Staaten als primäre Subjekte des internationalen Rechts eigentlich nicht nur ein Erstzugriffsrecht, sondern auch ein Alleinzugriffsrecht auf Gesetzgebung innehaben, geben sie mit der Freigabe und Unterstützung der Selbstregulierung diese Position ohne Not auf. Wird sonst eine vormals staatlich ausgeführte Materie an die Privatwirtschaft ausgelagert, erfolgt das nur mit gleichzeitigem Auf- und Ausbau von Regulierung und Kontrolle. Dass das im Bereich der Ausübung von Gewalt, ja sogar Staatsgewalt, nicht nur versäumt, sondern bewusst nicht durchgeführt wurde, führt zu einer Erstarkung der Machtposition von PMCs gegenüber den Staaten. Es wird schwer, gegen das Argument der bestehenden (und nach eigenen Angaben funktionierenden) Selbstregulierung eine harte Regulierung zu etablieren. Je länger dieser Zustand andauert, desto mehr wird er sich verfestigen. PMCs haben sich damit im Schatten des Fokus auf die Anti-Söldner:innenkonvention etablieren können. ${ }^{98}$

Solange die strategischen Gründe für den Einsatz von PMCs weiterbestehen, ist von einer weiteren Nichtregulierung auszugehen. Die strategischen Gründe bestehen aber fort, da es keine Regulierung gibt. Damit 
dreht sich die Regulierungsfrage im Kreis. Profitieren werden davon weiterhin die PMCs, die ihre Dienstleistungen frei anbieten können und Strafverfolgung und Haftung nicht ernsthaft befürchten müssen.

Wenn bestimmte militärische Dienstleistungen ausgegliedert werden, erlangen PMCs auch die Kontrolle über die Implementation der Dienstleistung. Sie gewinnen damit einen Erstzugriff auf die Ausübung von physischer Gewalt und Entscheidungsgewalt über die Ausübung, ein vormals den Staaten vorbehaltenes Privileg. Verstärkt wird das Problem, da einige Leistungen komplett an PMCs ausgelagert werden, sodass sie (zumindest kurzfristig) nicht mehr von Staaten allein ausgeübt werden können. ${ }^{99}$ Die Militärunternehmen machen sich so als ihre eigenen Gesetzgeber und Ausführende unersetzlich. Durch die Vergabe von Verträgen ohne Ausschreibungen und ohne Kontrolle der Einhaltung der Vertragsbedingungen können sich die erhofften Kostenvorteile auch nicht ergeben. ${ }^{100}$ Auch wenn die Unternehmen im Sinne und Auftrag von Staaten handeln, können durch die angesprochenen Umgehungsstrategien demokratische Beteiligung und Kontrolle ausgehebelt werden. Die eigentlich dominante Position der Staaten als Gesetz- und Auftraggeber wird durch die selbstverschuldete Lage deutlich geschwächt.

\section{Verhältnis Staat-Betroffene}

Im Verhältnis zwischen einsetzenden Staaten und Betroffenen muss festgestellt werden, dass eine staatliche „Flucht ins Private“ gelingt. Würden anstatt PMCs Soldat:innen handeln, wären die einsetzenden Staaten über die Staatenverantwortlichkeit haftbar. Diese ist für PMCs aber schwer herzustellen, da eine Zurechnung des privaten Verhaltens trotz der Beauftragung schwer ist und wohl nur eine potentielle due diligence-Verpflichtung in Betracht kommt. Besonders prekär wird die Situation durch Vereinbarungen zwischen Entsende- und Aufnahmestaaten, dass letztere PMCs nicht strafrechtlich verfolgen werden. Staaten können also militärische Dienstleistungen outsourcen - zu Lasten der von der Gewalt Betroffenen.

Private Militärunternehmen agieren im Bereich des staatlichen Gewaltmonopols. Dabei werden sie als legitime Akteure unter der Prämisse wahrgenommen, dass sie das Gewaltmonopol achten und stärken. ${ }^{101}$ Diese Prä-

99 Leander (Fn. 17), S. 117.

100 Isenberg, U.S. Grand Strategy (Fn. 95), S. 23-27; siehe oben, S. 353f.

101 Leander, Security Dialogue 2010, 467 (481). 
misse ist aber nicht (mehr) zu halten. Durch die großen Regulierungslücken, die damit einhergehende Möglichkeit der Selbstregulierung und der daraus resultierenden faktischen Straffreiheit konnten PMCs ihre begrenzte Kontrolle über die Ausübung von physischer Gewalt zu einer Entscheidungsgewalt ausbauen, die eine genaue Trennschärfe zu Kompetenzen im Bereich der Staatsgewalt vermissen lässt. Das stellt demokratische Prozesse, das Gewaltmonopol und den Schutz von Opfern vor teils massive Probleme.

\section{Ausblick}

Der schlichte Mangel an anwendbarem und handlungsbegrenzenden Recht und das Desinteresse an der Verfolgung des wenigen, welches anwendbar wäre, bedingen gemeinsam den derzeitigen Status von PMCs. Auch Selbstregulierung kann keine Abhilfe schaffen. Daraus ergeben sich massive Konsequenzen für den Menschenrechtsschutz Betroffener und das Potential zu dauerhaften Verschiebungen der Machtstrukturen zwischen privaten Unternehmen und Staaten im Bereich des Gewaltmonopols.

Diese Probleme sind nicht einfach zu lösen und scheinen perpetuiert. Rufe nach internationaler Regulierung wurden bisher nicht erhört, aus den dargelegten Gründen ist mit einer baldigen Initiative nicht zu rechnen. Aus dem Arsenal des internationalen Rechts könnten die UN Principles on Business and Human Rights eigenständig angewendet werden, stellen jedoch für PMCs auch kein hard law dar. Auch die bestehenden Initiativen zum Umgang von bewaffneten nichtstaatlichen Akteuren könnten Ansatzpunkte zur Behandlung von PMCs bieten. ${ }^{102}$ Eine Stärkung der lokal zuständigen Rechtsverfolgungsbehörden durch nationale Gesetzgebung oder (finanziellen) Mitteln, wäre eine Möglichkeit, die keines internationalen Konsens bedarf. Zudem kann an eine stärkere Einwirkung auf die Selbstregulierung durch die Zivilgesellschaft und Staaten hingewirkt werden, sodass die Selbstregulierung kein zahnloser Tiger bleibt. Das würde aber gleichzeitig die Selbstregulierung erstarken lassen, was einer echten gesetzlichen Regulierung abkömmlich wäre.

102 Statt vieler: Joint Statement by independent United Nations human rights experts on human rights responsibilities of armed non-State actors, https:/www.ohchr.org/EN/NewsEvents/Pages/DisplayNews.aspx?'NewsID=26797\&LangID=E (letzter Aufruf am 1.4.2021). 